\title{
Applications of the Cracow X-Ray Microprobe in Tomography
}

\author{
J. BieleCKI ${ }^{a}$, S. BożeK ${ }^{a, b}$, J. LeKKI $^{a}$, Z. StachurA ${ }^{a}$ And W.M. KWiAteK ${ }^{a}$ \\ ${ }^{a}$ The Henryk Niewodniczański Institute of Nuclear Physics, Polish Academy of Sciences \\ Radzikowskiego 152, 31-342 Cracow, Poland \\ ${ }^{b}$ The Jagiellonian University Medical College, Department of Pharmacokinetics and Physical Pharmacy \\ Medyczna 9, 30-688 Cracow, Poland
}

\begin{abstract}
A nuclear microprobe at the IFJ PAN in Cracow has found numerous applications in different fields of research, mostly in biophysics, medical sciences, geology, and material research. In order to extend the research possibilities, a new X-ray microprobe was constructed. This new microprobe consists of three experimental lines dedicated to: (i) X-ray irradiation of biological specimens, (ii) elemental analysis of samples by micro X-ray fluorescence or total reflection X-ray fluorescence methods and (iii) computer microtomography. In this paper the computer microtomography line was described. The line consists of an open type Hamamatsu L9191 X-ray tube with microfocusing to about $2 \mu \mathrm{m}$, a high resolution X-ray sensitive CCD camera, and a precise goniometer composed of six piezoelectric motors. Depending on the required X-ray energy, the Hamamatsu tube is used with $\mathrm{Ti}, \mathrm{Mo}, \mathrm{Ag}$, or W targets. A small focus size and short focus-to-object distance enable to obtain images of samples with a magnification of more than $1000 \times$ and resolution of the order of $2 \mu \mathrm{m}$. The computer microtomography measurements are carried out using home developed codes combined with commercial software. Details of the microprobe construction and preliminary results of the computer microtomography experiments are presented.
\end{abstract}

PACS numbers: 87.59.-e, 87.57.Q-

\section{Introduction}

The nuclear microprobe at the IFJ PAN in Cracow has found numerous applications in different fields of research, mostly in biophysics, medical sciences, geology, and material research. In order to extend the research possibilities, a new X-ray microprobe has been constructed. This new microprobe consists of three experimental lines dedicated to: (i) X-ray irradiation of biological specimens, (ii) elemental analysis of samples by micro X-ray fluorescence $(\mu \mathrm{XRF})$ or total reflection X-ray fluorescence (TXRF) methods and (iii) computer microtomography (CMT). As the microprobe use is not restricted to CMT experiments only, either soft or hard $\mathrm{X}$-ray beams are required. Therefore, as an X-ray source, an open type tube (Hamamatsu L9191) was chosen with extremely small X-ray emitting spot size, a wide range of accelerating voltage and exchangeable anodes. In this paper the CMT line has been described. The line is built using the modules described in the following sections.

\section{Radiation source and hardware}

Experimental line dedicated to CMT experiments consists of a source of radiation, an X-ray sensitive CCD camera, and a precise goniometer. The source-to-detector distance is usually set as $500 \mathrm{~mm}$ while source-to-sample distance can be changed from $1 \mathrm{~mm}$ to almost the source-to-detector distance, ensuring a wide range of magnification tuning.

An open type, microfocusing X-ray tube Hamamatsu L9191 with exchangeable transmission targets [1] serves as a source of X-rays.

Four different targets made of titanium, molybdenum, silver, and tungsten are provided in order to tune the X-ray energy depending on specimen's density. Acceleration voltage can be changed in the $20-160 \mathrm{kV}$ range, whereas the tube current may be set up to $200 \mu \mathrm{A}$. Radiation is emitted from a small spot (less than $2 \mu \mathrm{m}$ in diameter) into a cone (120 degrees opening angle), through $0.5 \mathrm{~mm}$ thick beryllium output window. This geometry of the X-ray beam is used for tomography experiments without using any additional focusing devices. Cone beam geometry entails that projections have to be acquired with equiangular separation over $360^{\circ}$.

In order to evaluate the spatial resolution of the system a test with microfabricated pattern has been carried out. The pattern, produced nanolithographically by Xradia Ltd., is a circle composed of wedges $16 \mu \mathrm{m}$ wide at the outer part of the circle and narrowing down to $0.5 \mu \mathrm{m}$ for smaller radii [2]. Figure 1 presents image of the pattern obtained with the use of the X-ray microprobe, while Fig. 2 shows the relation between the acceleration voltage and the obtained focal spot size.

A size of the X-radiation emitting spot was measured to be about $2 \mu \mathrm{m}$ for accelerating voltage of the tube 


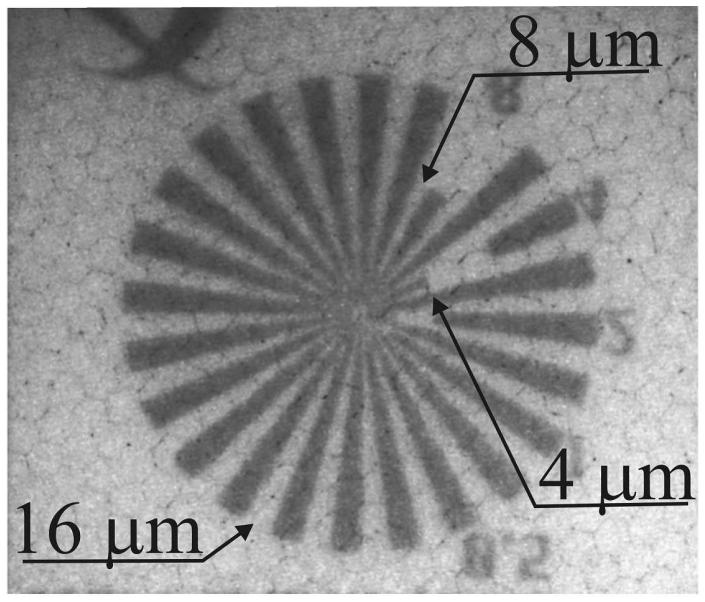

Fig. 1. X-ray image of the microfabricated pattern. The outside cogs have the size of $16 \mu \mathrm{m}$, cogs on the middle circumference have the size of $8 \mu \mathrm{m}$, and are narrowing down to $0.5 \mu \mathrm{m}$ for the smallest radii.

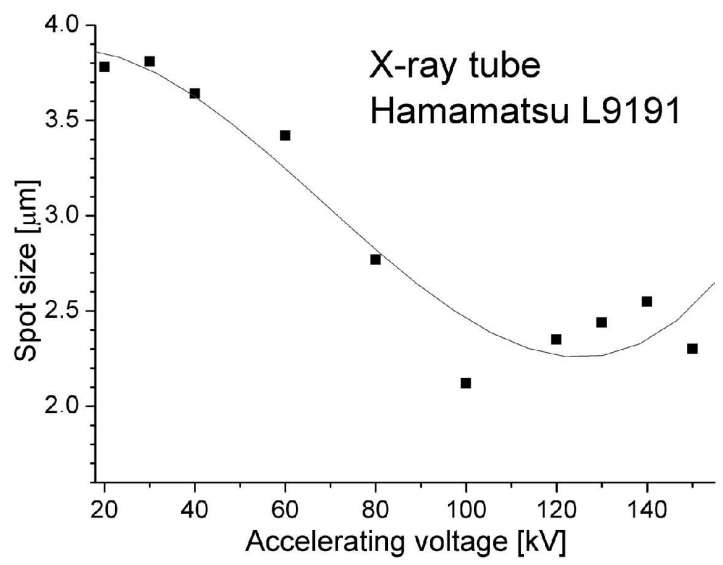

Fig. 2. The focal spot size as a function of the acceleration voltage.

above $100 \mathrm{kV}$. However, the resolution is deteriorating for lower voltage being about $4 \mu \mathrm{m}$ at $20 \mathrm{kV}$ [3].

The X-ray tube flux has been measured with the use of XR-100CR (Si pin photodiode) and XR-100T-CdTe (CdTe diode) detectors. Figure 3 presents the normalized number of Ti $K_{\alpha}$ photons $(4.5 \mathrm{keV})$ as a function of acceleration voltage. The total number of photons of energy higher than $5 \mathrm{keV}$ emitted in $1 \mathrm{~s}$ per $1 \mathrm{sr}$ is showed in Fig. 4. Statistical errors have not been marked, because they are insignificantly small when compared to errors caused by instability of the tube current. Intensity of $\mathrm{Ti}$ $K_{\alpha}$ lines is two orders of magnitude lower than intensity of bremsstrahlung radiation. The use of such polyenergetic beam in computed tomography experiments can lead to appearance of artifacts (see Sect. 4).

Positioning and rotating of a sample is accomplished with the use of six piezoelectric motors. Three of them are used to move the sample in the $x-y-z$ directions.

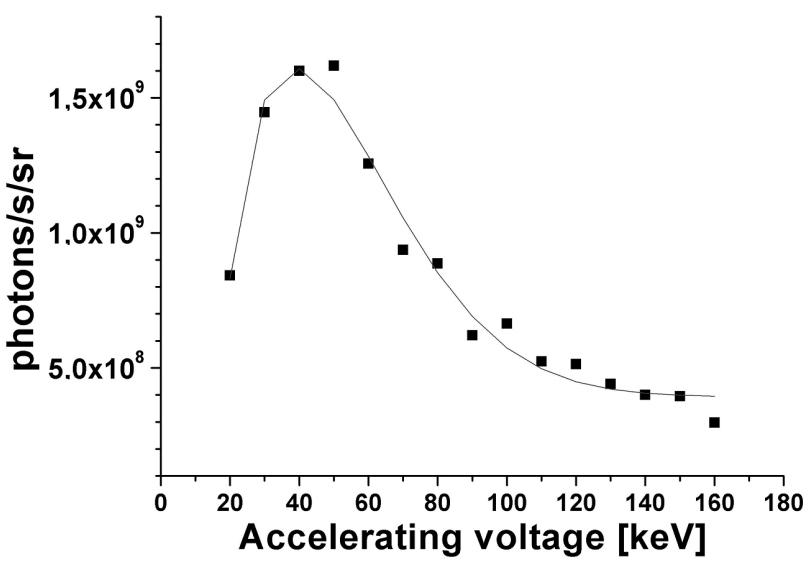

Fig. 3. The number of $\mathrm{Ti} K_{\alpha}$ photons emitted in $1 \mathrm{~s}$ per $1 \mathrm{sr}$ as a function of accelerating voltage.

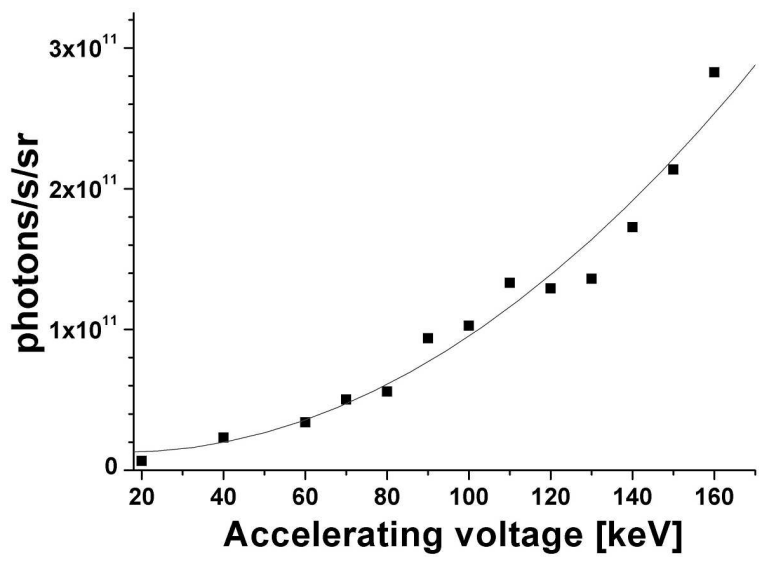

Fig. 4. The number of photons of energy higher than $5 \mathrm{keV}$ emitted in $1 \mathrm{~s}$ per $1 \mathrm{sr}$ as a function of accelerating voltage.

Another two motors enable tilting the rotation axis and the last one serves as a precise rotator. The actuators are stacked vertically and form a precise goniometer. Maximum load for these devices is $100 \mathrm{~g}$. The accuracy of translation for linear motors reaches half micrometer, whereas angular precision for rotator is about $0.1^{\prime \prime}$. Three main motors are equipped with resistive readout encoders which enables the "on-line" measuring of the specimen position.

Image acquisition is accomplished with Photonic Science X- ray sensitive CCD camera [4]. The sensor is composed of $4008 \times 2670$ pixels, readout at $10 \mathrm{MHz}$ frequency, followed by digitization of the CCD signal to 12-bit accuracy (4096 gray levels). Each pixel has a size of $14.7 \mu \mathrm{m}$ in diameter. The CCD sensor is optically bound to a tapered fibre-optic. The fibre ending has been covered with the X-ray scintillator (gadolinium oxysulphide doped with terbium, approximately $10 \mathrm{mg} / \mathrm{cm}^{2}$ ). The thickness of the scintillator is optimized to register $\mathrm{X}$-rays of energies in the range of 5 to $35 \mathrm{keV}$. The image 
readout is carried out by means of a commercial frame grabber card installed in a personal computer.

In order to check linearity of the detector used for image acquisition in CMT experiments, the detector response as a function of tube current has been determined. The output signal was measured in the flat field mode by increasing the intensity of the X-ray beam up to the maximum signal amplitude value measurable by the CCD, with avoiding saturation. The X-ray tube was equipped with Ti target and set at $70 \mathrm{kV}$, varying its current from 1 to $10 \mu \mathrm{A}$. Source-to-detector distance was set to $410 \mathrm{~mm}$. The output signal was first averaged over 5 frames and then averaged over a region-of-interest of about $4000 \times 2500$ pixels to minimize the effects of non-uniformity in the illumination. The response signal is highly linear in a wide range of the absorbed dose of X-rays, with R-square $=0.9997$.

The full block diagram of the setup and the associated electronics is presented in Fig. 5. For image acquisition and experiment control a solution based on two PCs has been chosen. The first computer is dedicated to control the conditions of the experiment (monitoring and setting the X-ray tube parameters and readout and setting the sample position), while the second PC is used for data acquisition and analysis as well as for image reconstruction. Communication between the two PCs is accomplished with a use of TCP/IP protocol.

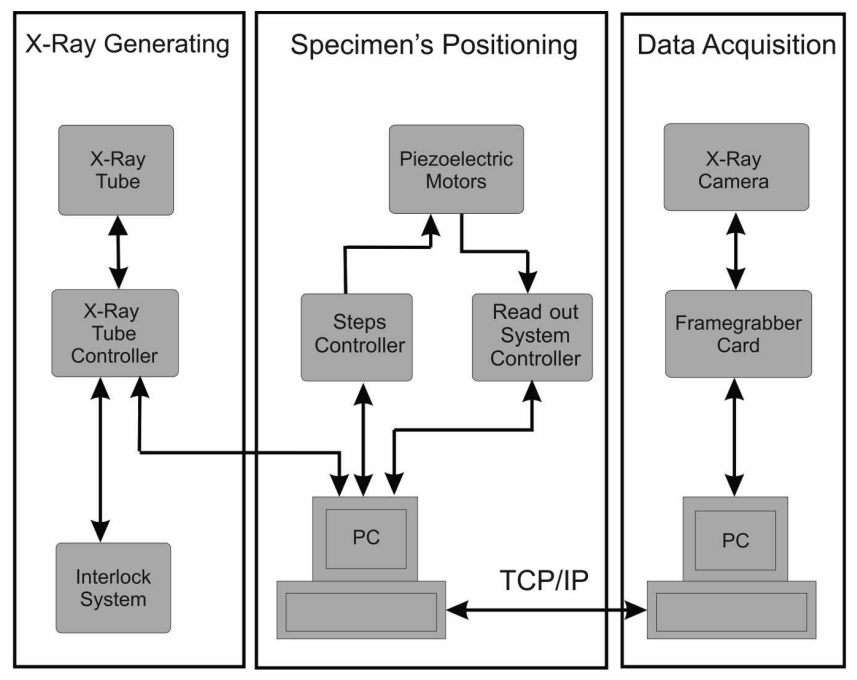

Fig. 5. Electronics associated with CMT experiments.

Hardware modules used in the CMT experiment are functionally divided into three groups. The first one, responsible for $\mathrm{X}$ radiation generation, consists of the X-ray tube, its controller, and the interlock system assuring the radiation safety. The X-ray tube controller is remotely controlled by a PC via the RS232 port. The two other PC serial ports are connected to units controlling positioning and movement of the measured specimen. The goniometer, composed of six inertial piezoelectric motors, is controlled by a steps controller connected to the PC.
Actual position of the sample is registered in the computer through the read out system controller, connected to the resistive encoders incorporated into piezoelectric motors. The last group of modules forms the data acquisition track. These are the X-ray sensitive CCD camera and a frame grabber card installed in a slot of the second PC.

\section{Software system and data flow}

Software system used in the CMT measurements is based on a few complementary computer programs a home developed code as well as few commercial packages. Experiment is controlled by a home-made program written in $\mathrm{C}+$ which is linked with the Image Pro Plus package [5]. Projections, flat fields, and dark images are stored in the TIFF format on the PC hard disks. These files are the input data for the reconstruction package.

The Octopus package based on filtered back projection algorithm [6] is used for slices reconstruction. Reconstruction time of tomography image from a data set consisting of 500 projections each of $512 \times 512$ pixels, reconstructed in the cone beam geometry using the normal quality setting takes about $30 \mathrm{~min}$ at the $\mathrm{PC}$ computer with AMD Athlon X2 Dual Core 64 bit CPU and 2 GB RAM memory.

The $3 \mathrm{D}$ visualization and analysis is accomplished with the Avizo package [7]. Figure 6 shows a diagram of the data flow in $\mathrm{CT}$ experiments.

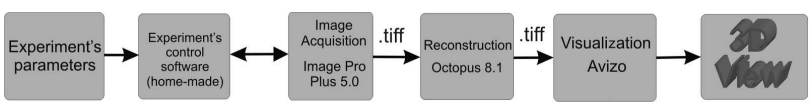

Fig. 6. Scheme of software system used in CT measurement.

\section{Results of first CT experiments}

In order to evaluate the performance of the system as well as the performance of the reconstruction process several tomography experiments were carried out with centimeter and millimeter size object.

Figure 7 presents reconstructed tomography images of a transistor and its photo. On reconstructed images, a thin wire inside the transistor is clearly visible. Images were reconstructed from 400 projections each of $500 \times 333$ pixels. Projections were obtained with a use of the polyenergetic cone beam emitted from the X-ray tube equipped with the $\mathrm{W}$ target. Acquisition time of a single projection was $20 \mathrm{~s}$. The reconstruction process was accomplished with a use of filtered back projection algorithm implemented in the Octopus package [8]. The reconstructed image consists of $500 \times 500 \times 311$ voxels, $10 \times 10 \times 15 \mu \mathrm{m}^{3}$ size each one.

In order to check the possibility of imaging geological samples a few tests were carried out. Figure 8 shows tomography images of a sample of andesite rock and its 


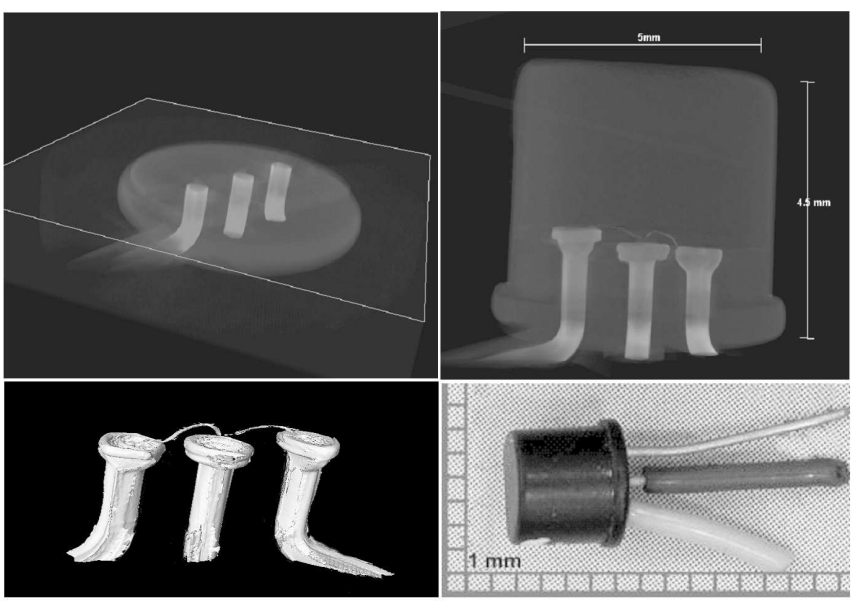

Fig. 7. Tomographic images and the photo of a transistor.

photo. Reconstruction was obtained from 500 projections $(744 \times 666$ pixels $)$, acquired with a use of $\mathrm{W}$ target. Integration time of single projection was about $30 \mathrm{~s}$. Tomography images contain $744 \times 744 \times 500$ voxels. Single voxel size is equal to about $10 \times 10 \times 10 \mu \mathrm{m}^{3}$.Additionally, a reconstruction of the $2 \mathrm{~cm}$ long shell is shown in Fig. 9.

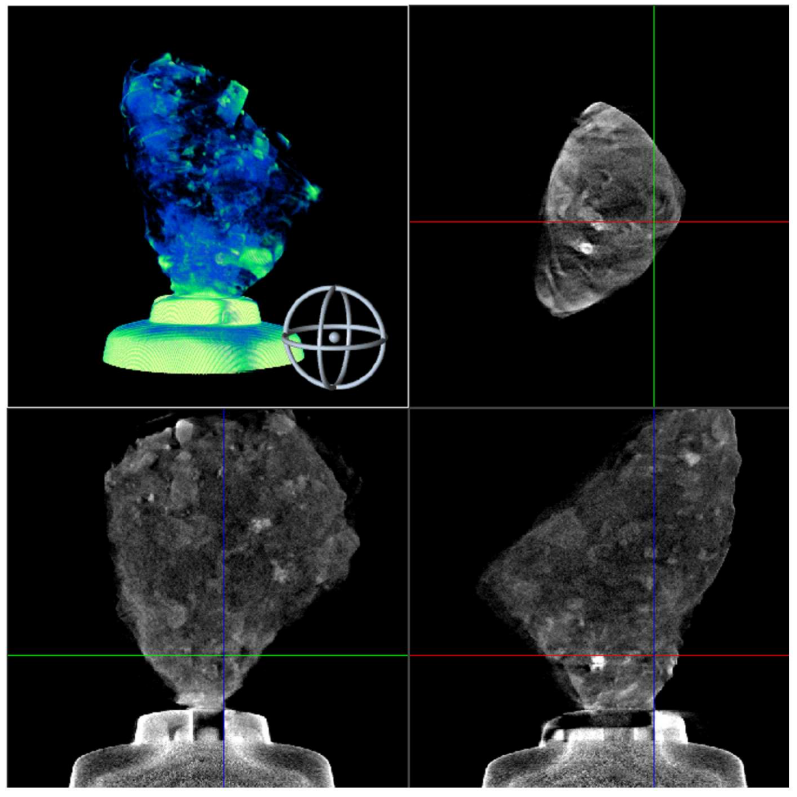

Fig. 8. Tomography images of the sample of andesite rock (sample size $\approx 1 \mathrm{~cm}$ ).

\section{Discussion of artifacts}

Images are reconstructed from projections, which are subjected to noise and errors. Any error of projections' measurement influences the reconstructed images. In reconstructions, several types of artifacts can be observed.

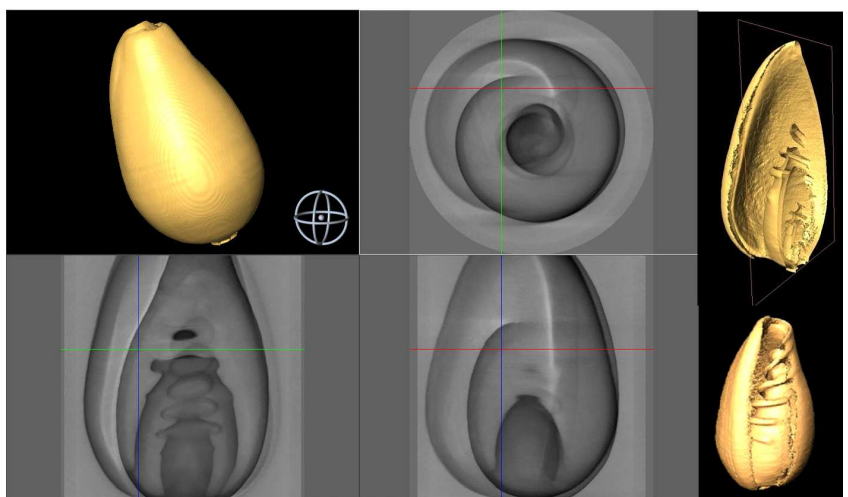

Fig. 9. Tomography images of a shell. The image contains $437 \times 437 \times 600$ voxels reconstructed from 500 projections $(744 \times 666$ pixels $)$, acquired with the use of $W$ target. Sample size $\approx 1 \mathrm{~cm}$.

The first artifact type is caused by mechanical instability of the specimen during the experiment. Vibrations cause errors in projections and, in consequence, distortions in the reconstructed slices. To avoid this artifact it is necessary to use the exposition time as short as possible. Also, the overscan technique, providing data redundancy and averaging inconsistencies in the projection dataset, was used in order to minimize motion artifacts.

The second type of artifacts is related to so-called "rings". They appear as an increase or decrease in the image intensity values along a circle (or part of a circle), centered at the center of rotation of the system. The artifact is caused by different sensitivity of some pixels in the CCD matrix.

First method used by us to reduce the ring artifacts is a "flat field correction". This is a simple method of error correction that is accomplished by taking the image of the beam without the positioned sample before and after projection acquisition. The data are next used to sample image normalization. This method can correct to some degree errors caused by inhomogeneous sensitivity of pixels in the CCD camera as well as local inhomogeneities in the X-ray beam intensity. Besides flat field images correction, the so-called "dark images" were also collected, in order to correct dark current of the detector. Dark images are subtracted from the projection images and the open beam images.

However, the above approach cannot remove ring artifacts entirely. Therefore the next step is the post-processing of tomography images. This process is accomplished with a use of the algorithm of sinogram processing during the reconstruction implemented in the Octopus package.

Ring artifacts are recognized in the sinograms as vertical lines from top to bottom. Reduction of this artifact is made in the following way: for each sinogram the average of each column is calculated and compared to the average value of the nearest neighbor columns. When deviating by more than a chosen level, the pixel values in the col- 
umn are multiplied by a correction factor so that the average of the column becomes equal to the median of the averages of its neighbors. This procedure performs better than the commonly used method of applying a low pass filter in the perpendicular direction which introduces unnecessary blurring [9]. Figure 10 presents reconstructed slice with, and without ring filtering.

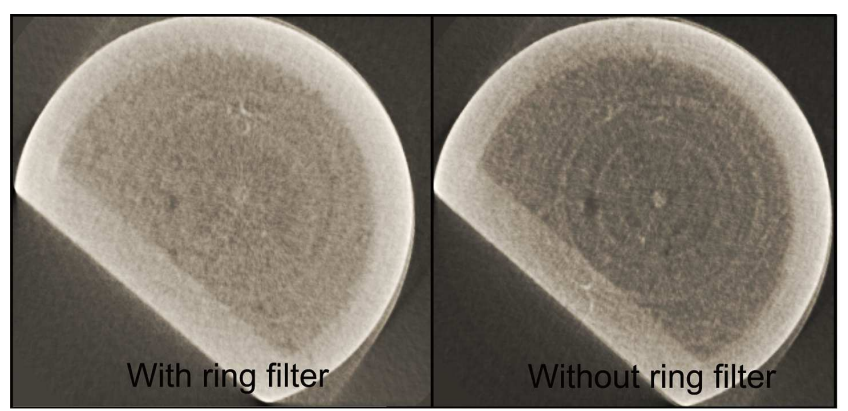

Fig. 10. Example of reconstructed slice images with and without the ring filtering.

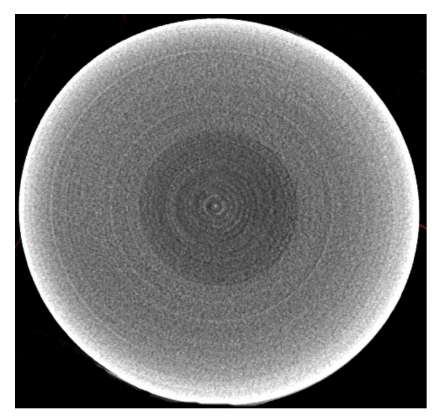

Fig. 11. Appearance of the beam hardening artifact in the image of reconstructed slice.

A use of polyenergetic radiation can cause an appearance of the "beam hardening" distortions (Fig. 11). As the polyenergetic beam of X-rays passes through an object, the mean energy of the beam increases, because the lower energy photons are more absorbed than the higher energy ones. This effect can also lead to artifacts (see [10]). The most noticeable effect is the cupping of reconstructed samples. A reconstructed density value in the center of the sample is seemingly lower than at the sample edges. The simplest way to reduce the beam hardening artifacts is to use the "pre-hardening" filter absorbing low energy part of the X-ray beam, such as $0.5 \mathrm{~mm}$ thin filter made from aluminum or copper. The negative side of this method is the reduction of the X-ray beam flux. Complementary to the filter method is the use of the beam hardening correction algorithms implemented in the Octopus package. This software offers two correction methods for a two-phase object (like material + air): an advanced beam hardening correction algorithm and a faster polynomial correction [11].

\section{Conclusions}

The above examples illustrate the operational possibilities of the system that may be currently considered as ready for routine use. However, several issues still require attention and improvements, as reported above. Currently, the main goal is the optimization of focal spot size by the tube focus alignment in order to improve spatial resolution of the facility (see [2]) and the reduction of artifacts in CMT experiments. In particular, it is planned to implement a mixed hardware/software mode of operation, enabling the reduction of rings artifacts by random, small movements of the sample between subsequent projections.

\section{Acknowledgments}

Purchase of hardware and software for the X-ray microprobe was supported by the Foundation for Polish Science and Technology, grant No. 222/FNiTP/119/2005. Partial support from the MP0601 project of COST is also gratefully acknowledged.

\section{References}

[1] The Hamamatsu website, http://www.hamamatsu.com.

[2] The Xradia website, Resolution and Calibration Targets, 2008.

[3] S. Bożek, J. Bielecki, J. Baszak, H. Doruch, R. Hajduk, J. Lekki, Z. Stachura, W.M. Kwiatek, Nucl. Instrum. Methods Phys. B, submitted for publication.

[4] The Photonics Science website, http://www.photonic-science.co.uk.

[5] The MediaCybernetics website, http://www.mediacy.com.

[6] The XRayLAB website, http://www.xraylab.com.

[7] The Avizo program website, http://3dviz.mc.com/products/avizo.asp.

[8] M. Dierick, B. Masschaele, L. Van Hoorebeke, Measur. Sci. Technol. 15, 1366 (2004).

[9] Manuel Dierick, Ph.D. Thesis, Gent University, 2005.

[10] J.F. Barrett, N. Keat, RadioGraphics 24, 1679 (2004).

[11] J. Vlassenbroeck, M. Dierick, B. Masschaele, V. Cnudde, L. Van Hoorebeke, P. Jacobs, Nuclear Instrum. Methods Phys. Res. A 580, 442 (2007). 